\title{
Overthrowing the dictator: a game-theoretic approach to revolutions and media
}

\author{
Hubert Janos Kiss ${ }^{1,2}{ }_{(\mathbb{D}} \cdot$ Ismael Rodríguez-Lara $^{3}(\mathbb{D} \cdot$ \\ Alfonso Rosa-García 4
}

Received: 13 January 2016 / Accepted: 8 June 2017 / Published online: 16 June 2017 (C) The Author(s) 2017. This article is an open access publication

\begin{abstract}
A distinctive feature of recent revolutions was the key role of social media (e.g. Facebook, Twitter and YouTube). In this paper, we study its role in mobilization. We assume that social media allow potential participants to observe the individual participation decisions of others, while traditional mass media allow potential par-
\end{abstract}

Hubert Janos Kiss: MTA KRTK_-Research fellow in the Momentum (LD-004/2010) Game Theory Research Group.

\begin{abstract}
We are grateful for comments and suggestions from participants at the MKE Conference in Budapest and the SING 2014 Meeting on Game Theory in Krakow, and seminar participants at the Corvinus Game Theory Seminar (Budapest), the Universidad de Murcia and Universidad de Malaga (Spain). Hubert Janos Kiss gratefully acknowledges financial support from the Spanish Ministry of Economics under research project ECO2014-52372, the János Bolyai Research Scholarship of the Hungarian Academy of Sciences and the National Research, Development and Innovation (NKFIH) under the project K 119683. Ismael Rodriguez-Lara and Alfonso Rosa-Garcia acknowledge financial support from the Spanish Ministry of Economics under research projects ECO2014-58297-R, ECO2013-45698-P and ECO2016-76178-P.
\end{abstract}

$凶 \quad$ Ismael Rodríguez-Lara

I.Rodriguez-Lara@mdx.ac.uk

Hubert Janos Kiss

kiss.hubert.janos@krtk.mta.hu

Alfonso Rosa-García

arosa@ucam.edu

1 MTA KRTK KTI, Budaörsi út 45, 1112 Budapest, Hungary

2 Department of Economics, Eötvös Loránd University, Lágymányosi Campus, Budapest 1117, Hungary

3 Department of Economics, Middlesex University London, Hendon Campus, The Burroughs, London NW4 4BT, UK

4 Facultad de Ciencias Jurídicas y de la Empresa, Universidad Católica San Antonio de Murcia, Campus de Los Jerónimos, s/n Guadalupe, 30107 Murcia, Spain 
ticipants to see only the total number of people who participated before them. We show that when individuals' willingness to revolt is publicly known, then both sorts of media foster a successful revolution. However, when willingness to revolt is private information, only social media ensure that a revolt succeeds, with mass media multiple outcomes are possible, one of which has individuals not participating in the revolt. This suggests that social media enhance the likelihood that a revolution triumphs more than traditional mass media.

"We use Facebook to schedule protests, Twitter to coordinate, and YouTube to tell the world."

Anonymous Cairo Activist

\section{Introduction}

Coordination failures occur when individuals do not act in unison to achieve an outcome that would benefit them. Examples include, among others, poverty traps, economic cycles or inefficient conventions (Masiliūnas 2017; Cooper and John 1988; Adsera and Ray 1998; Morris 2014). In these settings, individuals may benefit from choosing an action that changes the status quo, but it would require the coordination of a sufficient number of individuals to do so. Revolutions are also akin to these setups. Citizens that protest might change a bad regime if the number of participants is above a certain threshold. One issue to be considered is that part of the population might be unwilling to participate in the revolt. In this paper, we look at the effect of communication technology (mass or social media) to overthrow a dictator when the majority wants to change the regime, but there is heterogeneity of types and part of the population is unwilling to change the status quo.

In the last years, there have been many mass mobilizations that attracted considerable public attention internationally. The mass protests that started the uprisings against the regimes in the Arab world, the demonstrations of the Indignados movement in Spain, the Occupy Wall Street movement or the Hong Kong's Umbrella revolution are the most prominent examples. A distinguishing feature of these events was the omnipresence of social media (especially, Facebook, Twitter and YouTube). Many scholars (e.g. Castells 2011; Edmond 2013; Manacorda and Tesei 2016; Enikolopov et al. 2016) wonder how these new information technologies affect social movements. Do they help to overthrow autocratic regimes more easily than the former technology?

Social media affect the evolution of protests in various ways. First, social media provide access to alternative information about the true state of the economy and the government's performance. This information may be limited under mass media, especially when the government controls it. Social media help to inform audiences around the world about the unfolding of the events, attracting international attention and provoking diplomatic pressure. Finally, social media offer an easy, quick and inexpensive means of communication that facilitates the spread of information among the participants. This, in turn, may help to foster coordination, a key factor to achieve the goals of any movement. One interesting insight along these lines is one by Daron Acemoglu. When asked about overthrowing dictators he pointed out the following: "It 
is a question of coordinating people's beliefs, I need to know if other people agree with me and are willing to act. What really stops people who are oppressed by a regime from protesting is the fear that they will be part of an unsuccessful protest. When you are living in these regimes, you have to be extremely afraid of what happens if you participate and the regime doesn't change."1 Descriptions of the recent revolutions seem to highlight the importance of social media in efficient mobilization. Individuals fear that not enough people will go to the protests, so uncertainty about the turnout is a major obstacle. When mass media (e.g., TV or radio) report about attendance, then the audience does not know the reasons why individuals did not go. In social media these reasons can be revealed. For instance, Ghonim (2012) mentions the existence of opinion polls online to find out why people decided (not) to go to the protest. The comments on Facebook help also to get insight into the decision-making of others and serve to raise spirits. Ghonim describes that he was surprised to see among the supporters persons that he did not expect to see. All this together helps to "break the barrier of fear" (Ghonim 2012) and allows individuals know that they are enough and together they can bring down the dictator.

Although social media seem to have a notable effect on mobilizing the masses, we lack a formal model that attempts to capture its effects on mobilization. In this paper, we use game theory to show how this technology may help to change the status quo in an autocratic regime. Our starting point is that willingness to participate in the protests depends on the perceived costs and benefits of participation. Arguably, heading out onto the streets implies the costs of facing tear gas, rubber bullets and potential arrest and incarceration. Benefits involve the perceived gains in participating in an uprising that may bring about a better future, provided it succeeds. The probability of success is highly related to the number of participants. Yet, when a potential protester decides whether to participate, possibly she has only a vague idea about if sufficient other people will participate. Different types of media may affect these beliefs (and the resulting mobilization) in diverse ways.

To investigate the effects of social media on mobilization, we study how individuals' decisions to participate in the revolution are affected by two different communication technologies: mass and social media. We posit that when an individual obtains information through mass media then she gets to know the actual state of the revolution in that moment, whereas when informed via social media she is able to observe the sequence of decisions leading up to that state. For instance, when searching in Twitter the individual gets the last conversations about the topic, and by scrolling down the page she is able to see all previous tweets about it, the use of hashtags indeed facilitates this task. In Facebook, the users may comment on the events and all previous comments can be read. When people chat in a group with Whatsapp, the complete talk is recorded in the smartphone of each participant. We suppose that tweets, chats and comments are informative about the individuals' decision to join the protests or

\footnotetext{
1 The New York Times, February 17, 2011, "Web Solutions Applied to the Problem of Toppling Autocrats", retrieved from http://www.nytimes.com/2011/02/18/world/americas/18iht-letter18.html?_r=0 on July 14, 2014. Manacorda and Tesei (2016) posit a similar argument and discuss the possible benefits of information: 'Knowledge, albeit imperfect, of others' likelihood of participating can, in particular, foster individuals' willingness to participate, and lead to the emergence of protests in equilibrium, an outcome that would not result in a world where individuals act atomistically." (page 3).
} 
to stay at home. As stated by participants in the Hong Kong's Umbrella revolution, "If your friends ask you to join the protest, they just Whatsapp you: 'Hey, I am going, are you?' Then you quickly decide." (Parker 2014). By contrast, when TV or radio inform about the state of a given event, the precise history remains hidden, only aggregate information about the turnout is reported. ${ }^{2}$

We rely on these distinctive features of mass and social media and model the problem of revolution as a coordination problem. We suppose that there are two groups in the society. One of them consists of individuals who want to overthrow the dictator (willing individuals). The another one is composed of individuals who do not want to change the regime but prefer the status quo (unwilling individuals). We assume that there are enough willing individuals to bring about a change and overthrowing the dictator is the socially efficient outcome. That is, if all willing individuals revolt, then the dictator is toppled. However, if the number of protesters (those willing individuals who actually head onto the streets) falls short of a critical mass, then the dictator remains in power and may punish those who participated in the failed revolt.

We assume that individuals choose in sequence whether or not to revolt. Before making her decision, each individual is informed about the state of the revolution. The information that an individual receives depends on the communication technology. As discussed above, mass media allow individuals to learn how many people have already chosen to participate (i.e. the actual state of the revolution), whereas individuals observe each of the past decisions (e.g. the precise history) when information is channeled through social media.

First, we show that when the type of individuals (that is, if she is willing to revolt or not) is public information, willing individuals take part in the revolution in case of both communication technologies and, thus, the dictator is overthrown. Interestingly enough, it is not necessarily the case when there is no communication technology and thus individuals receive no information about the state of the revolution. If willing individuals believe that other willing individuals will not revolt, then it is possible to have an equilibrium outcome where nobody revolts. This, in turn, shows that the mere existence of communication technologies (i.e., mass or social media) can facilitate that social movements achieve their objective by shrinking the set of beliefs, compared with the case in which no information is disclosed.

Second, we study a more realistic setup in which the type of individuals is not observed (i.e. it is private information). Although it is common knowledge in our model that there are enough willing individuals in the society to change the regime, the type of communication technology becomes relevant in this setup. We show that mass media do not necessarily enable willing individuals to organize themselves efficiently. Thus, depending on the perceived costs and benefits, willing individuals might choose not to revolt in equilibrium. However, successful revolution is the unique equilibrium outcome when individuals use social media, independently of the severity of punishment that protesters may suffer if they fail to overthrow the dictator.

2 There are features of social media that foster spreading information quickly and to a wide audience. For instance in the case of Twitter "retweeting" allows to repost a content referencing to the source of the content; "trending topic" highlights content that an in-built algorithm considers collectively relevant. 
This result shows that communication through social media facilitates that revolutions succeed more than when communication is channeled through traditional mass media.

Although our comparison of mass and social media suggests that they are competing communication technologies, our results are aimed at answering the question about how social media enhance mass media's ability to mobilize individuals. The results gleaned from the empirical evidence support the idea that citizens perceived social media to be very important during the Arab Spring (Howard et al. 2011; Lang and De Sterck 2014), including the Tunisian revolution (Marzouki et al. 2012) or the protests in Egypt (Attia et al. 2011; Azab 2012; Lim 2012; Tufekci and Wilson 2012). Hence, microblogging activity (Qin et al. 2016) or the amount of tweets (Acemoglu et al. 2014; Steinert-Threlkeld et al. 2015) might have been related to the incidence and the intensity of protests. There is indeed a causal relationship between social media and mobilization according to Manacorda and Tesei (2016) and Enikolopov et al. (2016). Manacorda and Tesei (2016) study how coverage of mobile phone signals affected the occurrence of protest and individual participation on the whole African continent between 1998 and 2012. ${ }^{3}$ They show that mobile phones promoted protests and helped mass mobilization during economic downturn. Enikolopov et al. (2016) show that the penetration of VK, the dominant Russian online social network, affected protest participation in a series of protest during 2011. More concretely, a 10\% increase in penetration increased the probability of a protest by almost $5 \%$ and the number of participants by roughly $20 \%$.

Section 2 reviews briefly the relevant literature. Section 3 presents our model. We derive our theoretical results in Sect. 4, where we also discuss the application of our model, by presenting two examples in which we assess the differences between mass and social media in yielding different outcomes in a revolution. We discuss some caveats of our model and possible extensions in Sect. 5. Section 6 concludes. All the proofs are relegated to the Appendix.

\section{Literature review}

In this section first we show why observability of actions and social networks (that are the basis of social media) are important in the evolution of revolts. Then we go over the most relevant and recent theoretical papers on revolutions and the corresponding literature on coordination games.

Our interpretation of the process why an individual joins a protests follows the rationalist view that the citizens assess the costs and benefits of participating in a revolt (see, for instance, Goldstone 2001 and the references therein). ${ }^{4}$ Social networks play an important role at several stages of the process. Passy (2003) claims that social media create social networks that are important in community creation, in connecting

\footnotetext{
3 The authors point out that in Africa there is a lack of fixed phone line and high-speed Internet cabling, so generally mobile phones provide the access to the Internet and social media.

4 There are other views on protests that emphasize the role of identity and social-psychological factors (see for instance, Klandermans 1984). In revolts, both rational and non-rational elements play a role, in this paper we focus on the former ones.
} 
the prospective participants and also in the decision-making, since individuals use the information coming from the social network to anticipate and evaluate the potential costs and outcome of participation. Gunitsky (2015) and Battaglini (2017) highlight also the effects of social media in allowing individuals to communicate their information. Importantly, the decision to join the revolution depends also on the intentions and action of other participants. If many other citizens are expected to join, then the revolution is likely to succeed and this makes participation more attractive. In the opposite case, staying at home may be the optimal decision. Both mass and social media enable individuals to form beliefs about the turnout at the protest. Our approach aims to show that social media promote better mobilization, because from an individual's point of view they give more accurate information about previous decisions and they allow that subsequent individuals observe the decision. The fact that decisions can be observed and may affect the behavior of others relates our work to models of informational influence; i.e., herding and informational cascades (e.g., Banerjee 1992; Bikhchandani et al. 1992, 1998). However, in these models there is a clear best choice (e.g., which restaurant or product is better) and individuals have some private information about it. Individuals update their signals upon observing the decision of others, thus it may be rational to follow others to choose the payoff-maximizing alternative. In our setting, there is heterogeneity of types in that willing and unwilling individuals have different preferences. Relevant to our setting, willing individuals should revolt in equilibrium only if the revolution turns out to be successful, thus a coordination problem is embedded in our framework. These features make our paper divert from the literature on informational influence.

Turning to theoretical models of revolutions, a common point in most of these papers is that the regime can be overthrown if enough citizens participate in an uprising. The studies differ mainly in (i) what types of individuals they assume, (ii) if the individuals have different willingness to revolt, (iii) the channels of coordination (e.g., what can be observed?), and (iv) the regime's role. De Mesquita (2010) assumes the existence of a vanguard, a continuum of citizens with varying antigovernment sentiment and a passive regime. It is a pure coordination game with simultaneous moves after the vanguard chose the level of costly violence. The vanguard uses violence and it is informative about the discontent in the society and individuals sensing the increased dissatisfaction are more likely to join the revolution. ${ }^{5}$ Individuals do not observe other individuals' decision to join the protest, therefore the role of media is disregarded in this model, which has multiple equilibria: with and without successful regime change. Chwe (2000) is the closest paper to ours in spirit. He assumes two types of individuals: willing (those who want change and are ready to go to the streets) and unwilling (those who stay at home). In his model, the social network allows individuals to communicate their private type (e.g., Gunitsky 2015; Battaglini 2017). Chwe characterizes the minimal sufficient networks that make coordination feasible among willing individuals, regardless on the prior beliefs about the willingness of the others. He shows the importance of cliques (a subset of individuals where everybody is

\footnotetext{
5 Barbera and Jackson (2016) argue that demonstrations may help in providing information to enable revolutions. See also Ginkel and Smith (1999) for a model in which a group of willing individuals choose whether or not to revolt before the mass public.
} 
linked to everybody else). In our setting the main difficulty comes from what a willing individual believes when observing that somebody stays at home (i.e., she can be an unwilling individual who does not want to change the regime or a willing individual that prefers not to revolt). Our approaches differ in that we do not allow individuals communicate their willingness to participate using the network, but to observe the action of others. Chwe (2000) also looks at the minimal sufficient network for the revolution to succeed, while we assume that the network is complete and study how different sorts of information (mass vs. social media) might help to overthrow the dictator. ${ }^{6}$ Ellis and Fender (2011) is also akin to our approach in that observing other individuals' behavior is possible. This helps to make inferences about the information that other individuals have about the state of the world. The revolution is successful if an information cascade forms and poor agents rebel. We do not have a herding model as Ellis and Fender (2011) and contrary to their model individuals in our setup revolt only if they can be sure that the uprising will succeed. Edmond (2013) studies the revolution also as a coordination game in a global games framework. Citizens are ex ante identical and then they receive noisy signals about the regime's strength from several media outlets, but not about the decisions of other citizens. Lang and De Sterck (2014) show that mobilization is successful only if protesters are enthusiastic enough and their action is visible.

In the previous papers, revolution is modeled as a coordination problem that might be overcome in different ways. In the models without observing other citizens' decisions (De Mesquita 2010; Edmond 2013) observing correlated signals makes a revolt potentially successful. In the models where observing other individuals plays a role (Ellis and Fender 2011; Lang and De Sterck 2014) there is only one type of individuals and if enough of them decide to revolt, then the dictator is overthrown. Our model attempts to capture the uncertainty involved in mobilization, resorting to unwilling individuals whose choice is always the same. Arguably, their presence makes coordination difficult, since a willing individual who observes somebody staying at home does not know if it is due to an unwilling citizen or a willing one who decided not to participate in the revolt. This feature makes our paper divert from the literature on global games (Carlsson and Van Damme 1993; Morris and Shin 2003; Angeletos et al. 2007) where introducing (and not removing) some uncertainty might help in efficiently resolving the multiple equilibria problem, yielding a unique equilibrium prediction.

\section{The model}

We study in a model how different communication technologies determine the outcome of a revolt. Suppose a finite set of individuals, $N=\{1,2, \ldots, n\}$ and a dictator. Each individual chooses an action $a_{i} \in\{r, s\}$ where $r$ means "revolt" and $s$ "stay at home". Each individual $i$ is either of type $\tau_{i}=w$ (willing to revolt) or $\tau_{i}=x$ (unwill-

\footnotetext{
6 The network structure in Chen et al. (2016) is such that individuals receive information about about the strength of the regime, and then communicate to each other the informativeness of the rumor. Kiss et al. (2016) is also related to Chwe (2000) in that they look at the minimal sufficient network, but in line with our modeling choice they assume that the network allows for the observability of actions.
} 
ing). Willing individuals are ready to participate in protests, unwilling individuals are reluctant to do so. We denote by $W$ the amount of individuals that are willing to revolt, i.e., $\#\left\{i: \tau_{i}=w\right\}=W$, where $W \in(0, n)$.

Individuals decide in a sequence. Let the type vector $\tau=\left(\tau_{1}, \tau_{2}, \ldots, \tau_{n}\right)$ denote the sequence of individuals. ${ }^{7}$ The set of sequences of length $n$ with $W$ willing citizens is given by

$$
\Upsilon^{n, W}=\left\{\tau: \#\left\{\tau_{j} \in \tau: \tau_{j}=w\right\}=W\right\}
$$

There are $\left(\begin{array}{c}n \\ W\end{array}\right)$ possible type vectors and any of them is selected with equal probability. $^{8}$

We suppose that the index of the individual $(i \in N)$ corresponds to her position in the sequence of decisions. The utility of each individual $i$ depends on her type and the outcome of the revolution. The revolution is successful if at least $t$ individuals decide to revolt (i.e., $\#\left\{j \in N: a_{j}=r\right\} \geq t$ ), otherwise the dictator will remain in power. We follow De Mesquita (2010) and assume that the value of the threshold is common knowledge. ${ }^{9}$ We consider the case in which $W \geq t$ and $W$ is common knowledge, so that individuals know that there are sufficient people willing to revolt. ${ }^{10}$ Changing the regime is assumed to be the socially efficient outcome (as it will also be clear from the payoffs). Although it is common knowledge that there are sufficient willing individuals in the society to change the regime, and overthrowing the dictator is efficient, the change requires coordination. This, in turn, depends on the individuals' expected costs and benefits of participating in the revolt.

Let $a_{i}$ be the action chosen by individual $i$ and let $a=\left(a_{1}, a_{2}, \ldots, a_{n}\right)$ be the profile of actions. A willing individual that decides to stay at home $\left(a_{i}=s\right)$ will receive utility $u_{w, s}$. If the willing individual decides to participate in the revolt, the utility will depend on whether the revolt succeeds $\left(u_{w, r, R}\right)$ or fails $\left(u_{w, r, F}\right)$, where $u_{w, r, R}>u_{w, s}>u_{w, r, F}$ is assumed. ${ }^{11}$ In words, willing individuals' utility is highest when they participate in a successful revolution $\left(u_{w, r, R}\right)$. If they stay at home, they derive less utility $\left(u_{w, s}\right)$, although the smallest utility is derived when individuals take part in a revolution that is defeated. The payoff $u_{w, r, F}$ can then be interpreted as the

\footnotetext{
7 Abusing somewhat the notation, $\tau$ denotes a sequence of individuals, but also the set of individuals in the sequence.

8 This, in turn, implies that we study any possible configuration that may occur in equilibrium in a pregame, in which individuals have to choose when to decide. We are not aware of any paper that studies how the sequence of decision in a revolt is determined. We discuss this issue in Sect. 5.

9 Schelling (1978) and Granovetter (1978) use models with individual thresholds to study problems that involve collective action. In Edmond (2013) or Angeletos et al. (2007) there is uncertainty about the threshold. In Chwe (2000) each person has an individual threshold.

10 If $W<t$, then it is clear that individuals do not revolt in equilibrium.

11 Note that in the utilities, the first subscript refers to the type of the individual, the second to the action that she undertakes, whereas the third one indicates the outcome. $R$ represents a successful revolution, while $F$ denotes that it has failed.
} 
punishment that the dictator imposes on protesters who participate in a revolution that fails, suffering this punishment being the potential cost of participation. ${ }^{12}$

We assume that unwilling individuals will not participate in the revolt (whatever reasons they might have). The utility of an unwilling individual that revolts $\left(u_{x, r}\right)$ is therefore assumed to be smaller that the utility of an unwilling individual that stays at home $\left(u_{x, s}\right)$. This, in turn, implies that it is always optimal for an unwilling individual to stay at home. Interestingly, the presence of these individuals complicates the coordination among willing individuals. A willing individual who observes somebody staying at home does not know if it is due to an unwilling citizen or a willing one who decided not to participate in the revolt.

Given our payoffs, the first best is achieved when willing individuals coordinate and overthrow the dictator. The reason is that unwilling individuals' utility is not affected by the outcome of the revolt, whereas willing individuals are better off if the uprising is successful.

Our paper is an attempt to show how different communication technologies may affect the outcome of the revolution. Individuals decide in sequence whether or not to revolt and they have information about past decisions that is available to individuals depending on the communication technology as follows:

- No technology Individuals do not have any information on previous choices when deciding.

- Mass media technology Individuals have aggregate information about the actions that have been already taken (e.g., number of predecessors that decided to revolt and stay at home). This represents a situation in which individuals obtain information through radio or television about the state of the revolution before making their own decision.

- Social media technology Individuals observe the individual action of each predecessor. This means that individual $i$ knows exactly which action was chosen by each of her $i-1$ predecessors. This represents a situation in which individuals obtain information through Facebook or Twitter (or any other social media), in which individuals may observe the exact history of previous decisions.

To formalize the different communication technologies, let $\varphi_{i}$ denote the information that individual $i$ has. When no communication technology is available, then $\varphi_{i}=\left\{\tau_{i}\right\}$; i.e., individuals only know their own types, but nothing about other individuals' decisions. Mass media technology implies $\varphi_{i}=\left\{\tau_{i}, \rho_{i}, i-\rho_{i}-1\right\}$ where $\rho_{i}$ represents the number of individuals who have decided to revolt up to individual $i\left(\rho_{i}=\#\left\{a_{j}=r, j<i\right\}\right)$; i.e., individuals know the amount of predecessors who decided to participate in the revolt, and then the number of individuals who decided to stay at home. In the spirit of Lohmann (1993, 1994a, b) we assume that each previ-

12 The utility of staying at home may depend on whether the revolution triumphs or not. A successful revolt may bring better life to a willing individual who by staying at home avoids the costs of the revolution. Thus, there may be free-riding issues at stake as well (see for instance Lohmann 1993). Although these are interesting questions (and promising venues of future research), we deliberately disregard this issue to focus on the coordination problem embedded in the above payoffs. In this regard, our payoffs generate a game that resembles the classic stag-hunt situation, although the presence of unwilling individuals complicates the analysis. 
ous decision (ordered according to the position) is observed under social media. The available information then is $\varphi_{i}=\left\{\tau_{i},\left\{a_{j}, \forall j<i\right\}\right\}$.

In Fig. 1, we depict a reduced extensive-form representation of the game generated by each communication technology in a simple society of $n=4$ individuals in which $W=3$ of them are willing to revolt. We name it reduced form because we have simplified the representation by drawing only one of the four branches that would follow each of the type vectors. At the beginning of the game, nature selects at random one of them. In the four possible type vectors willing citizens are represented by black circles and the unwilling one by a white circle. We assume that individuals only observe actions but not types, so individuals are represented by grey circles in the rest of the tree. ${ }^{13}$ We derive in Sect. 4 the theoretical predictions of our model both when types are observed and when they are private information.

At the top of Fig. 1, we represent the case without communication technology in which the individuals decide without knowing the decisions of their predecessors (i.e., they only know the position, but nothing else). As a result, all nodes at a given position belong to the same information set. Then, we depict the case of mass media, in which individuals only know the number of predecessors that already revolt. For instance, imagine that the fourth individual is informed that two individuals have chosen to revolt (and, consequently, one stayed at home). She is not able to distinguish if the sequence was $(r, r, s),(r, s, r)$ or $(s, r, r)$, hence the information that the individual has is compatible with three possible sequences of decisions. Finally, we draw at the bottom of Fig. 1 the case of social media, in which individuals are able to identify perfectly the sequence of decisions; i.e., the information sets are singletons. ${ }^{14}$

Note that the game becomes more sequential as we move from the case without communication towards social media. In principle, the effect of more information is ambiguous. More information may be good for revolution since it allows individuals to make their actions more visible to the subsequent citizens; but at the same time, it could also foster coordination failure, e.g. if individuals find out that too many of their predecessors have chosen not to participate in the revolution. Note that they may observe many individuals staying at home because those observed citizens were the unwilling ones.

\section{Revolutions under different information structures}

This section derives three different predictions of our model. The first one shows that there are multiple equilibria when no communication technology is available, therefore it is possible to have the inefficient outcome in which individuals decide not to revolt. We highlight the importance of mass and social media in Sects. 4.1 and 4.2, where we show that willing individuals will revolt in the presence of any of

13 In Appendix $\mathrm{C}$ we represent the complete decision tree for the social media case. Note that in our model the social network is assumed to be complete. Siegel shows in several papers how in general the structure of the social network affects participation in collective action (Siegel 2009), the effect of repression on collective action (Siegel 2011) and how it interacts with mass media (Siegel 2013). Importantly, none of these papers studies the effect of different sorts of media on participation in revolts.

14 Recall, however, that types are not observable in the present setting. 

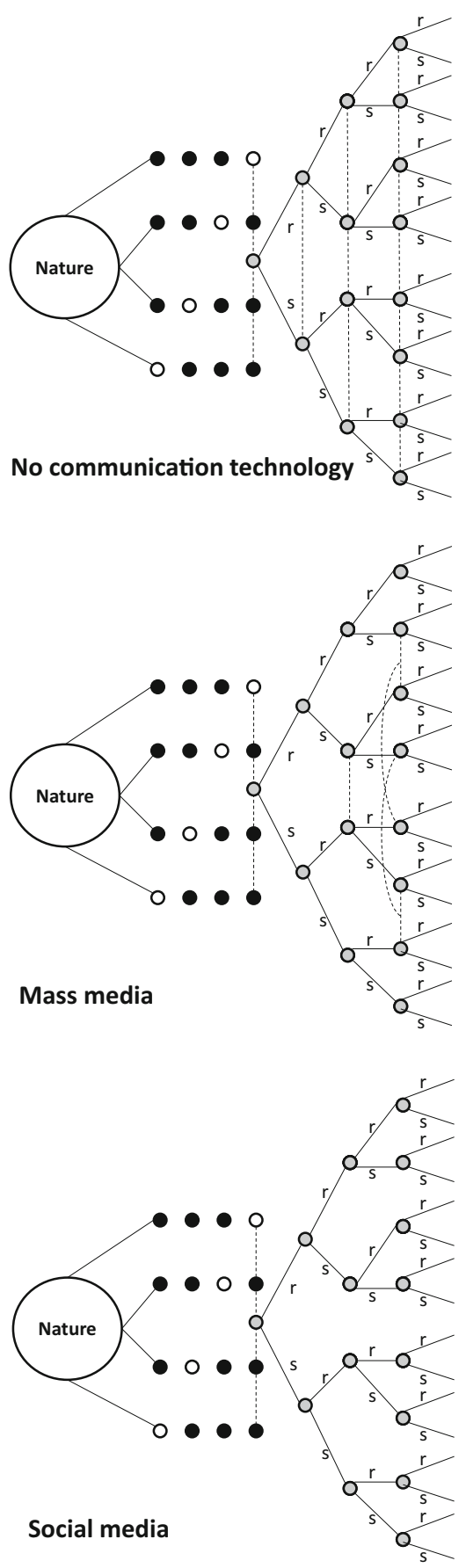

Fig. 1 Reduced extensive-form representation of a society with $n=4$ and $W=3$ 
the two communication technologies when types are public information. If types are unobservable, however, there are equilibria in which willing individuals decide not to revolt under mass media, whereas revolution will always succeed under social media.

The case without technology is our benchmark scenario. Our first result shows that when information about other individuals is not available (i.e., when decisions are taken without knowing what other individuals have done) the efficient outcome in which individuals revolt may fail to materialize, even if it is known that sufficient willing individuals exist.

Proposition 1 If no communication technology is available in the society, there are multiple equilibria.

This is in line with the usual result of multiplicity of equilibria in coordination problems. Since $u_{w, r, R}>u_{w, s}>u_{w, r, F}$, for the willing individuals it is optimal to revolt if the other willing individuals are revolting, while it is optimal to stay at home if nobody else is revolting. If a willing individual believes that the other willing individuals will participate in the revolution, then she best responds to this belief by participating as well. However, if they hold the opposite belief, then staying at home is the best response. In fact, there are two symmetric equilibria: one in which all willing individuals participate in the revolution; and another one in which no willing individual goes to the streets.

The previous result does not depend on whether type is a public information or not. However, when a communication technology is available this distinction becomes relevant as shown next.

\subsection{Type is public information}

It is instructive to see how the existence of information affects the outcome of revolts in a perfect information setup in which the willingness to revolt (that is, the type of individuals) is transmitted by the communication technology. In this setting, an individual who observes an action will know whether a willing or an unwilling individual took it. This can be the case, for instance, when the people willing to overthrow the dictator belong to the same social group (e.g. religious association, ethnic groups or social classes), so that individuals know the type of the people who have decided previously. For example, in Egypt the youth in general was unsatisfied with the regime, and so were also the Copts.

We model this situation by introducing the type of the predecessors in the information available to each individual. More specifically, the available information is $\varphi_{i}=\left\{\tau_{i}, \rho_{i}, i-\rho_{i}-1, W_{i}\right\}$ in the case of mass media, where $W_{i}$ denotes the amount of willing individuals up to (but excluding) individual $i$, that have already decided. ${ }^{15}$ As for social media, the assumption about publicly observed types implies that the

15 This is a valuable information since it also reveals how many willing individuals are left to decide. For instance, if there were many willing individuals who abstained from participating in the protests, then it is more probable that the total number of protesters will fall short of the threshold, so staying at home may be the best response. 
information of individual $i$ becomes $\varphi_{i}=\left\{\tau_{i},\left\{a_{j}, \forall j<i\right\},\left\{\tau_{j}, \forall j<i\right\}\right\}$. In this setting, both the type and decision of each preceding individual are observed.

Individual $i$ 's strategy is conditioned on the available information. It is defined as $\sigma_{i}: \varphi_{i} \rightarrow\{r, s\}$. Let $\Sigma=\{r, s\}^{n}$ be the game's strategy space, and let $\sigma \in \Sigma$ be a strategy profile, that is, $\sigma=\left(\sigma_{1}, \ldots, \sigma_{n}\right)$. Let $h_{i}$ be the history of decisions before individual $i, h_{i}=\left\{a_{1}, \ldots, a_{i-1}\right\}$.

We find that given a type vector the unique subgame perfect equilibrium with the two communication technologies is the one in which the revolution succeeds, and every willing individual chooses to revolt. In this case, both technologies generate the same behavior in equilibrium.

Proposition 2 If type is public information, every willing individual revolts in any subgame perfect equilibrium under both communication technologies.

\section{Proof See Appendix A.}

The rationale for this result is that a willing individual chooses to join the revolution if she observes that already $t-1$ individuals have revolted. Given this fact, a willing individual who observes $t-2$ people participating in the revolution decides to revolt if she knows that after her there is at least one more willing individual. Since predecessors' types are publicly observable, she can infer if there is a willing individual behind her. Iterating this reasoning, a willing individual decides to revolt when up to her sufficient willing individuals have chosen to do so and she anticipates that enough willing citizens behind her will follow suit. The conditions ensuring that this requirement is met at any position imply that all willing citizens choose to participate in the revolution.

As commented above, our assumption on predecessors' type being public information is plausible in environments where the people willing to overthrow the dictator can be associated to particular groups. Under these circumstances, it is likely that when individuals acquire information they know both the actions and the types of those who have already decided. In this case, the existence of any of the communication technologies guarantees that the revolution triumphs in our simplified environment. Arguably, the uncertainty about how many individuals would participate in a revolution is the main barrier and it makes most of the individuals who are discontented to stay at home. This uncertainty comes - at least partly-from the uncertainty about types that we consider next.

\subsection{Type is private information}

We study now the case where type is private information. Given the untrust and fear generated by dictators in repressive regimes, however, types cannot be observed, what makes the setup more plausible. As argued by several authors (e.g. Ginkel and Smith 1999; Kuran 1991, 1995), decision making in any revolution is clouded by a considerable amount of uncertainty. This uncertainty blurs the information about the public discontent due to several reasons, e.g. the lack of free press, falsified preference revelation to official public opinion polls or the presence of informants penetrating all layers of the society, among others. 
When type is private information, communication technologies only transmit to individuals the actions of predecessors. For the mass media technology, the information of individual $i$ becomes $\varphi_{i}=\left\{\tau_{i}, \rho_{i}, i-\rho_{i}-1\right\}$, so the amount of citizens of each type who have already decided is not known. In the case of social media, citizen $i$ 's information is given by $\varphi_{i}=\left\{\tau_{i},\left\{a_{j}, \forall j<i\right\}\right\}$. Hence, citizen $i$ cannot distinguish perfectly the type of her predecessors although she knows the exact sequence of decisions. Remember that observing that somebody revolts indicates unambiguously that she is of the willing type, since unwilling citizens always stay at home. However, since willing individuals may choose to stay at home, observing that someone has chosen not to participate in the revolt does not imply that she is unwilling.

Since types cannot be observed, we look for Bayesian Nash equilibrium. The following proposition highlights the importance of the communication technology. We find that under social media being truthful is the unique equilibrium profile; therefore, staying at home when an individual is unwilling and revolting when she is willing is the unique Bayesian Nash equilibrium. With mass media the revolution succeeds only when certain conditions are met.

Proposition 3 Consider the case where type is private information. Under the social media technology, the revolution always succeeds because each willing individual revolts in any Bayesian Nash equilibrium. Under the mass media technology,

- if $t<\left[\frac{n}{n-W+1}\right]+1$, each willing individual revolts and the revolution succeeds;

- if $t \geq\left[\frac{n}{n-W+1}\right]+1$, there are equilibria where the revolution is unsuccessful and nobody revolts for certain values of $u_{w, r, R} ; u_{w, s}$ and $u_{w, r, F}$.

Proof See Appendix B.

The logic behind our result is that "identifications of types" is possible under social media, but not under mass media. More specifically, any individual that observes a history of decisions under social media knows that all willing (unwilling) predecessors decided to protest (revolt) in equilibrium. With mass media, there may be situations in which willing individuals find it optimal to stay at home, thus a citizen that observes a history of decisions will only revolt if she is sure that there are enough willing to revolt behind her. Hence, a willing individual that observes $t-2$ revolts will always revolt in the case of social media (even if only one individual is left to decide). This is because the individual that observes $t-2$ protests can infer the types of the predecessors and knows that there is (at least) one willing individual to decide. In the case of mass media, an individual that observes $t-2$ revolts does not know how many willing individuals decided to revolt, thus she can only be sure that the revolution will triumph if she revolts and there are at least $n-W+1$ individuals left to decide. While these considerations are embedded in the second part of Proposition 3, the following example is aimed to clarify the mechanism why social media promote revolutions more than mass media. We consider the simple society of Fig. 1 in which there are $n=4$ individuals, and three of them are willing to overthrow the dictator $(W=3)$. We assume that the revolution will be successful in this society if and only if at least three individuals decide to revolt $(t=3)$. We then focus on the worst possible scenario and construct an equilibrium in which everyone stays at home under mass media. We show that there is a unique equilibrium where all willing individuals revolt in the case of social media. 
Example 1 Consider the case of $n=4, W=3, t=3 .{ }^{16}$ When a communication technology exists, the optimal decision of a willing individual in the last position is obvious. If she observes two people revolting $\left(\rho_{4}=2\right)$, then she revolts and the revolution triumphs. Otherwise she stays at home. The same is true for a willing individual in position 3 if $\rho_{3}=2$ (she best responds by revolting). As a consequence, a willing citizen in position 2 observing one revolting individual $\left(\rho_{2}=1\right)$ revolts as well, because she anticipates that if she decides to revolt, then the last willing individual (either in position 3 or 4 ) will follow suit. Thus, in any equilibrium a willing citizen revolts when $\rho_{4}=2 ; \rho_{3}=2$ or $\rho_{2}=1$, and stays at home when $\rho_{4} \in\{0,1\}$ or $\rho_{3}=0$. In these last cases, a willing individual knows that the revolution is doomed to fail, so she does not join. Thus, we are left with the following information sets for which a willing citizen's optimal action is not clear: $\rho_{1} ; \rho_{2}=0$ and $\rho_{3}=1$. In words, we do not know yet what a willing citizen does when she is the first to decide; when she is in the second position and observes no protester and when third in the sequence of decision and observes one protester. We show an equilibrium for the case of mass media where nobody revolts on the equilibrium path for some payoffs. Then, we show that it cannot be the case for social media.

Assume the existence of mass media and the following payoffs: $u_{w, r, R}=1, u_{w, s}=$ 0 and $u_{w, r, F}=-10$, that satisfy $u_{w, r, R}>u_{w, s}>u_{w, r, F}$. If nobody chooses to revolt in the previous information sets $\left(\rho_{1} ; \rho_{2}=0\right.$ and $\left.\rho_{3}=1\right)$ and acts optimally at the other information sets (i.e., revolts when $\rho_{4}=2 ; \rho_{3}=2$ or $\rho_{2}=1$, and stays at home when $\rho_{4} \in\{0,1\}$ or $\left.\rho_{3}=0\right)$ then, we end up in an equilibrium without revolution. To show that nobody has a profitable unilateral deviation, take the first individual. Her deviation consists in revolting instead of staying at home. This is profitable if the second individual is willing, because the first individual induces the second one to revolt as well by the arguments we have seen before. In this case, the revolution triumphs and the highest utility is obtained. When the second individual is unwilling (which has conditional probability $\frac{1}{3}$ ), then the proposed strategies imply that subsequent willing individuals will stay at home and the revolution fails. Therefore, the unilateral deviation is not profitable if and only if

$$
u_{w, s}>\frac{2}{3} u_{w, r, R}+\frac{1}{3} u_{w, r, F},
$$

which holds for the proposed payoffs. In the same vein, it is easy to check that if a willing individual in position 2 observes that nobody has revolted yet $\left(\rho_{2}=0\right)$, then she does not have a profitable unilateral deviation given the prescribed strategy. Consider now a willing individual in position 3 who observes that just one citizen has revolted $\left(\rho_{3}=1\right)$. According to the prescribed strategy, it is an off-equilibrium decision. Thus, consistent beliefs may include that a willing individual followed the strategy and one willing individual deviated and revolted. Those beliefs imply that she will be followed by the unwilling citizen. Along these lines, it is the inability of a

16 It follows from Proposition 1 that without any communication technology, there are two symmetric equilibria (in pure strategies). In one of them, the three willing individuals choose to revolt. In the other one, they choose to stay at home. This is the case because for a willing individual to revolt (stay at home) is the best response when she believes that the other willing individuals choose to revolt (stay at home). 
willing individual in position 3 to distinguish whether the first or the second individual stayed at home (and her pessimism) that makes it optimal for the willing individual in position 1 to stay at home. Note also that the equilibrium in which each willing individual participates exists if we simply consider a strategy profile that establishes that willing individuals should revolt when $\rho_{1} ; \rho_{2}=0$ and $\rho_{3}=1$.

Restrictions on off-the-equilibrium beliefs could eliminate the equilibrium in which citizens do not revolt. For instance, assume that the first individuals to decide were willing ones with certainty, so that the type vector is $(w, w, w, x)$. In this case it is profitable to deviate unilaterally from staying at home when a willing individual is the first to decide as given the best responses indicated above the revolution will triumph. In fact, our result suggests that repressive regimes may attempt actively to increase uncertainty about the public discontent (even in the form to hinder citizens to have detailed information about participation in protests) so that multiple equilibria, and hence potentially no protests remains an equilibrium.

Next, we show that under social media there is a unique equilibrium in which all willing individuals revolt and succeed in overthrowing the dictator. For a willing individual in the last position the previous arguments apply. Thus, upon observing that two other citizens have revolted (the order does not matter) she revolts as well, otherwise she stays at home. When in position 3, a willing individual joins the protest when observing two protesters. As a consequence, a willing individual in the second position, who observes that the first citizen decided to protest, will revolt as well, anticipating that the last willing individual (either in position 3 or 4) will join the protests as well.

As a next step, let us consider what happens if a willing citizen observes that the first citizen revolted, whereas the second one stayed at home. We denote this by $(r, s)$. By previous reasoning this individual can be sure that the second individual was the unwilling one (a willing individual in the second position would have joined the uprising upon observing that the first citizen revolted), so she knows that the last citizen is willing and anticipating her reaction to observing a history with two individuals revolting she decides to protest as well.

Given the previous argument, a willing individual in the first position chooses optimally to protest, since any history starting with a revolting citizen leads to a successful revolution (either if she is followed by a willing individual who protests herself or when followed by an unwilling one who stays at home, but then the next individual will join the protest and in any case the last willing individual will revolt as well). As a consequence, when observing that the first individual has stayed at home, willing citizens know that she must have been the unwilling one (i.e., individuals get to know that the type vector is $(x, w, w, w)$ and by backward induction they play the unique equilibrium in which all of them revolt). That is, since the willing individual in position 4 (3) revolts when observing two (one) previous revolts, the willing citizen in position 2 will revolt and thus the revolution triumphs, and the dictator is overthrown. Importantly, these arguments apply for any payoffs such that $u_{w, r, R}>u_{w, s}>u_{w, r, F}$. It is also worth noting that with social media the outcome is unique because individuals are able to distinguish the histories $(r, s)$ and $(s, r)$, while with mass media individuals may believe that (with some positive probability) the one who stayed at home is a willing individual. 
Proposition 3 establishes also a relationship between the threshold $t$ and the number of willing individuals in the society $W$ such that it is possible to construct an equilibrium where the revolution does not triumph in the presence of mass media. In the following example, we show the quantitative difference in the effectiveness of social media versus mass media in fostering revolutions. In particular, Example 2 shows that all willing citizens revolting can be achieved as an equilibrium with mass media only if a relatively low proportion of the society is required to overthrow the dictator.

Example 2 Consider the case of $n=10, W=7, t=5$. Our reasoning above implies that the revolution triumphs under social media. Mass media can also foment revolutions for any possible payoffs, but only if just a relatively low proportion of the society is required to participate in the revolution in order to overthrow the dictator. In particular, Proposition 3 states that the revolution succeeds for sure only if integer $\left[\frac{n}{n-W+1}\right]+1=3$ or less individuals are required to participate in the revolts. Since it is required that $t=5$ individuals revolt, it is possible that nobody revolts under mass media, even though more than two-thirds of the individuals are willing to overthrow the dictator in this society $(W=7) .{ }^{17}$

The intuition behind this result is the following. If an individual can be sure that the revolution succeeds, then she joins the protests. Whenever she may believe that with positive probability the revolution fails, it is possible to find a punishment that is sufficiently large to deter individuals from participating in the protests. A willing individual at position 7, 8, 9 or 10 cannot be sure that among the subsequent citizens there is a willing one, since possibly all of them are unwilling. Hence, if she revolts, in the worst case the number of participants increases only by 1 . Thus, a willing individual at these positions only revolts if she observes at least $t-1$ previous revolts. That is, she revolts if only one more revolting individual is needed to bring the uprising to triumph. At position 6, a willing individual knows that there is for sure one more willing individual behind her and she can convince her to revolt with certainty if she will observe $t-1$ revolts. Thus, the willing citizen at position 6 revolts if she observes $t-2$ revolts. A willing individual at position 5 knows that there are at least two willing citizens behind her, but she cannot make sure that both of them will revolt if she decides to revolt. This is the case because possibly the first of the willing individuals is at position 9 and then by previous arguments this citizen cannot be sure that there is another willing individual behind her. Hence, a willing citizen at position 5 knows that by revolting she can prompt for sure one more willing individual to participate, so she revolts if she observes $t-2$ revolts. The same reasoning applies to willing individuals at position 4 and 3 . A willing individual at position 2 knows that there are at least 5 willing individuals behind her. In the worst case, the next one is at position 6 and by previous reasoning even she knows that there is one more willing citizen behind her. So, at position 2 a willing individual knows that she can induce two more willing citizens. Hence, if she observes that $t-3$ individuals have already revolted, then she joins the protest. The same is true for a willing individual at position 1 . Note that a

17 The difference becomes larger if we scale up the numbers. If $n=100$ and $W=70$ then the revolution succeeds always with social media (for any $t \leq W$ ), while the protest triumphs with mass media only if $t \leq 4$ individuals are required to join. 
willing individual at position 1 cannot observe anybody revolting, so the threshold that enables a successful revolution is 3 or less citizens. Probably, the unwilling individuals are at the first 3 positions. However, rational actors understanding the game infer, that if with a threshold of 3 none of the first 2 citizens revolted, then it must be due to the fact that those individuals were unwilling. But it reveals at the same time, that there are enough willing individuals behind, so a willing individual at position 3 (or 4) will revolt. Note that with a threshold of 3 (or less) all willing individuals will revolt by the previous arguments.

With higher thresholds, the revolution may fail. In particular, suppose that payoffs are $u_{w, r, R}=1, u_{w, s}=0, u_{w, r, F}=-10^{100}$ and $t=4$. Hence, the dictator would punish very strongly (say, execute) the participants of a failed revolution. Consider the following strategy for willing individuals

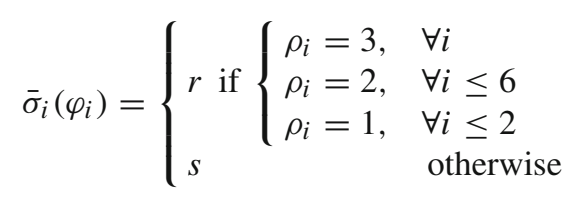

where $\rho_{i}$ is the number of participants that have chosen $r$ before individual $i$. A willing individual revolts at the information sets specified above. We have to prove that the strategy is optimal in the rest of information sets. Because a willing individual at position 1 observes nobody revolting, the proposed strategy profile states that she should stay at home. What if she deviates? Conditional on the first individual being willing, the probability that the second citizen is willing too is 6 out of 9 . Thus, with probability $\frac{2}{3}$ the deviation is successful and if the subsequent citizens follow the above strategy, then the revolution triumphs. However, with probability $\frac{1}{3}$ the next citizen is unwilling and she will stay at home. Then, if the subsequent willing individuals act according to the proposed strategy, the revolution fails. Given the payoffs it is easy to calculate that the expected utility of a willing individual in position 1 is negative, so the deviation is not profitable. The same argument can be applied for the rest of information sets to show that no deviation is profitable.

In our example, mass media communication guarantees that the revolt succeeds only if $t \leq 3$ individuals' participation is necessary to overthrow the dictator. To get an idea how our results affect mobilization, we scale up the numbers. In a society of 100 individuals in which 51 people are required to participate in the protests to change the regime, mass media facilitate a successful revolution (with all willing individuals revolting) for any payoffs only if it is known that all individuals $(W=100)$ are in favor of the revolution. If the required threshold were $t=49$, mass media would guarantee that the revolution succeeds only if it was commonly known that at least $W=99$ individuals are of the willing type. With social media, both $W=51$ and $W=49$ ensure that the revolt triumphs, respectively. Thus, mass media lead to a successful revolution only if there is a huge amount of people willing to participate in the protests, or if the dictator is very weak (the threshold is very low). In any other case, the dictator could implement a sufficiently high punishment $\left(u_{w, r, F}\right)$ so that revolts may not occur in equilibrium. 


\section{Discussion}

This paper has attempted to show that differences between the information transmitted by mass and social media can result in different outcomes in mobilization. Our research was prompted by the lack of a formal model that captures the importance of social media in mobilization during recent revolts. We argue that a potential channel for the success of the protests is that social media (e.g., Twitter or Facebook) provide a more detailed structure of information than mass media (e.g., TV or radio). More precisely, our model relies on the assumption that the exact history of past events can be followed using social media. Mass media provide also information about past decisions, but only in aggregate terms.

Theoretically, when the willingness to revolt is private information, our assumptions lead to a unique equilibrium with social media, where all discontented citizens revolt and the dictator is overthrown, while multiple equilibria occur with mass media, in one of them nobody revolts and the dictator remains in power. Our contribution to the existing literature is then to show rigorously that there is a qualitative difference between mass and social media in mobilization, the latter being able to promote mobilization more efficiently.

Our model uses numerous assumptions and simplifications that help tractability, but reduce its realism. Eventually, a lot of past decisions can be observed in any of the two communication technologies. Although this assumption is demanding, it has been used in Lohmann (1993, 1994a, b), who addresses questions of information aggregation and political action. In her models, individuals observe previous actions, but she does not study how different communication technologies affect coordination. In our setting, it is hard to believe that any individual is able to know exactly how many other individuals have joined the revolution through mass or social media; e.g., not all previous actions are observable through social media, since not everybody uses Facebook or Twitter. In addition, individuals may be biased when acquiring information online due to self-confirmation and polarization (Gentzkow and Shapiro 2011). In that regard, the assumption that the exact history of decisions has to be observed for the revolution to triumph can be relaxed. If there are enough willing individuals connected through social media (and this is common information), then all willing individuals will revolt and overthrow the dictator. We do not require that all previous decisions are observed to have a unique equilibrium in which the individuals revolt. If a group of citizens is connected through social media they will play a sequential game by observing the exact history of choices. Proposition 3 argues that the "identification of types" is possible in this setting. If this group of connected citizens contains at least $t$ willing individuals, then they will revolt in equilibrium and the revolution will triumph even if not all individuals are connected. Because types might be private information, we need a condition that guarantees that all citizens know that at least $t$ willing individuals will be connected. For example, when $N=100, t=25, W=50$, we do not require all the 100 citizens to be connected through social media but it is enough if 75 citizens are connected. These findings resemble the idea of cliques that guarantee coordination (Chwe 2000). One other way to relax the assumption that all individuals have to observe each other is to model a coordination problem among groups. Our findings could then be interpreted in terms of requiring that some groups in the society observe 
the action of others and coordinate. For instance, in Egypt several groups (e.g., Muslim Brotherhood, April 6 Youth Movement, soccer ultras) tried to coordinate the moves of their members for the mobilization to be successful. When applying our results to the coordination of groups, it does not seem too onerous to suppose that these groups know exactly what other groups are doing (e.g., Ginkel and Smith 1999; De Mesquita 2010; Barbera and Jackson 2016).

The other feature of our model that deserves some attention is the assumption that there are enough willing individuals to revolt. Most of the Arab countries (see chapter 3 in Filiu 2011) had a rather educated youth that was hit hard by unemployment and therefore in general was dissatisfied with the regime. Given the fact that the median age in these countries is around or less than 30, the youth represented a sufficient mass to change the system. Moreover, in some countries ethnic or religious minorities (e.g. the Copts in Egypt) also supported such aims. Thus, the assumption that there are enough willing individuals to overthrow the dictator (and this is common information) does not seem demanding, but one possible extension of our model is to consider that there is uncertainty about it. In such an alternative model, media might turn out to be informative about the feasibility of the revolution.

One other aspect of our setup is the assumption that individuals choose in an exogenously determined position. We are not aware of any paper that addresses this issue but one could try to endogenize such a decision. In the presence of media, individuals might want to postpone their decisions to get as much information as possible. Arguably, willing individuals may also want to choose early in the presence of media so as to signal their types and trigger the revolt, as discussed in De Mesquita (2010). The fact that positions are exogenously determined in our setting implies that our model is suitable for any possible sequence of decisions that could have been determined in a pre-game, in which individuals decided when to make their decision.

The issue of commitment problems is also worth discussing. One illustration of this problem would be supporting an event on Facebook, but not attending it. This consideration is not possible in our setting as individuals decide only once and cannot change their decision. In Egypt, page members took photos of themselves and posted them as a way to confirm participation. Giving the face can be seen as a way to overcome the commitment problem and rationalize our assumption, although we agree with De Mesquita (2010) that accounting for commitment problems is a fruitful area for future research.

A possibly even more severe issue is that media may be manipulated by the regime. During recent revolts, this does not seem to have been an issue in some countries. Filiu (2011) argues that for example in Egypt a relatively free press could evolve (e.g. journal Al-Masry al-Yom, TV station Dream) and Al-Jazeera, a media outlet with a high standard also broadcasts in many Arab countries. Thus, in principle, citizens could have a more or less accurate view of the situation. We focus on how the communication technology can favor revolts, and deliberately leave aside issues of manipulation or information about the performance of the government. Enikolopov et al. (2016) points out that fostering coordination by reducing its costs (which is ultimately the benefit of social media) might be more important to the successful protests than spreading information about the regime. We acknowledge, however, that autocratic regimes are aware of the importance of media. They tend to restrict access 
to social media, cutting off access to Facebook or Twitter, possibly because regimes fear the potential effects of further information. For instance, during the Arab spring several times they just shut Internet down for days. Regimes also actively monitor chats and forums and also develop fake websites to attract and identify potential opponents (see chapter 4 in Filiu 2011 or Gunitsky 2015). In the light of our findings, the dictator's decision of controlling the Internet or limiting the access to information and hence hindering coordination may receive some theoretical support. Along these lines, allowing the government to control the information, to make concessions to the protesters to appease them (Robinson and Acemoglu 2006) or taking into account the performance of the government (e.g. if the economy is doing worse than in other countries or if the inequality has risen) would help to enrich our setting. These issues may be also relevant from a policy perspective; e.g., what is the information that individuals should (not) receive for a revolt to triumph. ${ }^{18}$

Finally, we show in our setup that revolution is the unique equilibrium with social media. However, the mere existence of social media is not enough to bring about a regime change (see for instance, Dewey et al. 2012; Wolfsfeld et al. 2013). Although the demonstrations organized through Facebook were extremely successful in Egypt in January 2011, the April 6 Youth Movement failed to trigger mass protests using social media in 2008 in the same country. Similarly, in 2011, in Syria the "days of rage" protest was endorsed by 12,000 individuals online, but the turnout was low. Thus, there are other economic factors such as the level of education or the lack of opportunities that might help to explain why revolutions triumph (Campante and Chor 2012).

\section{Conclusion}

In this paper we have studied the differential effect of communication technologies on the outcome of revolutions in which overthrowing the dictator is assumed to be the socially efficient outcome. We distinguish mass and social media by the granularity of information that they provide. In particular, mass media supply an aggregate piece of information about the actual state of revolution, while when informed through social media individuals know the precise sequence of decisions that led to the actual state.

When individuals' types are public information, both communication technologies enable a successful revolution with certainty, a result that does not hold without communication technology. Hence, both mass and social media facilitate overthrowing the dictator. When types are private information, the sort of communication technology becomes relevant. With social media, the revolution succeeds in the unique equilibrium, whereas the revolution may fail with mass media except when the amount of people required to succeed is sufficiently low. If the punishment for participating in a failed revolution is sufficiently high, with mass media the no-revolution equilibrium may be sustained, but it is not true for the social media technology. As a result, our model shows that social media facilitate revolts more than mass media do.

18 Edmond (2013) analyzes in a different setup under which conditions a regime can be toppled if information is manipulated. See Chen et al. (2016) for a model in which citizens share rumors about the strength of the government and Pierskalla (2010) or Ginkel and Smith (1999) for a model in which the regime is an active player. 
Albeit the current study is motivated by revolts, the underlying situation is a coordination game with two types in which the uncertainty about types makes successful coordination more difficult. Our finding that the granularity of the information about previous decisions affects if the coordination is successful may be relevant in other socioeconomic environments that share the features of our game.

Open Access This article is distributed under the terms of the Creative Commons Attribution 4.0 International License (http://creativecommons.org/licenses/by/4.0/), which permits unrestricted use, distribution, and reproduction in any medium, provided you give appropriate credit to the original author(s) and the source, provide a link to the Creative Commons license, and indicate if changes were made.

\section{Appendix A}

Proposition 2 If type is public information, every willing individual revolts in any subgame perfect equilibrium under both communication technologies.

Proof The existence of the equilibrium is guaranteed by standard arguments. We show that in the unique subgame perfect equilibrium each willing individual revolts.

Let $\alpha_{i}=\#\left\{\tau_{j}=w: j \leq i \mid \tau_{i}=w, \tau\right\}$ be the position of a willing individual $i$ among the willing individuals in a given type vector $(\tau)$. Note that $\alpha_{i} \in\{1,2, \ldots W\}$, with $W>t$ and $\alpha_{i}$ is observable because types are public information

By backward induction, the last willing individual $\left(\alpha_{i}=W\right)$ revolts if at least $t-1$ other willing individuals decided to revolt. This is because he number of protesters reaches the threshold $t$ if she follows suit; i.e., the revolution will be successful. Otherwise, she decides to stay at home. The next to the last willing individual $\left(\alpha_{i}=\right.$ $W-1$ ) revolts if at least $t-2$ willing predecessors chose to revolt anticipating that then also the last willing individual will join in. Again, if the condition is not met, then she stays at home. This argument can be repeated for all willing individuals: the individual $\alpha_{i}$ revolts if at least $t-\left(W-\alpha_{i}+1\right)$ other individuals decided to revolt, otherwise she prefers to stay at home. As a consequence, the first willing individual $\left(\alpha_{i}=1\right)$ revolts even if she does not observe anybody revolting $(t-W<0)$. Therefore, the revolution succeeds.

Notice that the previous argument to find the unique subgame perfect equilibrium works for both types of communication technology mass and social media, thus Proposition 2 holds.

\section{Appendix B}

Proposition 3 Consider the case where types are private information. Under the social media technology, the revolution always succeeds because each willing individual revolts in any Bayesian Nash equilibrium. Under the mass media technology,

- if $t<\left[\frac{n}{n-W+1}\right]+1$, each willing individual revolts and the revolution succeeds;

- if $t \geq\left[\frac{n}{n-W+1}\right]+1$, there are equilibria where the revolution is unsuccessful and nobody revolts for certain values of $u_{w, r, R} ; u_{w, s}$ and $u_{w, r, F}$. 


\section{Proof Social media}

Let a history $h_{i}=\left\{a_{1}, a_{2}, \ldots, a_{i-1}\right\}$ be the set of actions chosen by predecessors of $i$. In the social media case, individual $i$ knows $h_{i}$ when she decides whether or not to revolt. Let $a^{i, *}$ be a set of actions for each individual $k>i$, such that action $a_{k}$ of each individual $k$ is a best response conditional on $h_{i+1}=\left\{h_{i}, a_{i}\right\}$, actions of its predecessors $(i+1, \ldots, k-1)$ according to $a^{i, *}$ and some consistent beliefs. Let $\pi_{j}$ be the payoff of $j$ given a profile of actions.

We define a truthful history as the one in which a willing individual $j<i$ strictly prefers to revolt in the information sets where it is observed that the individual stays at home (conditional on any possible best response by the individuals after $j$ and consistent beliefs), that is,

Definition 1 A history $h_{i}$ is truthful if and only if $\pi_{j}\left(r \mid \tau_{j}=w, h_{j} \in h_{i}, a^{j, *}\right)>$ $\pi_{j}\left(s \mid \tau_{j}=w, h_{j} \in h_{i}, a^{j, *}\right), \forall a^{j, *}, \forall j<i: a_{j}=s$.

We denote by $H^{t r}(k)$ the set of truthful histories which contain $k$ revolts at any position $i-1 \geq k .^{19}$ Truthful histories are those for which actions reveal the type, so anyone can make sure that only unwilling citizens stayed at home and the willing ones revolted. In a truthful history, consistent beliefs assign probability 0 to any type vector in which a willing individual would choose "stay at home" according to the history.

Lemma 1 If $\pi_{i}\left(r \mid \tau_{i}=w, h_{i} \in H^{t r}(k), a^{i, *}\right)>\pi_{i}\left(s \mid \tau_{i}=w, h_{i} \in H^{t r}(k), a^{i, *}\right), \forall k$ $>\hat{k}$, then $\pi_{i}\left(r \mid \tau_{i}=w, h_{i} \in H^{t r}(\hat{k}), a^{i, *}\right)>\pi_{i}\left(s \mid \tau_{i}=w, h_{i} \in H^{t r}(\hat{k}), a^{i, *}\right)$.

Proof The lemma assumes that when a truthful history containing more than $\hat{k}$ revolts is reached, for any possible continuation vector subsequent willing individuals will revolt. If it is the case, note that the only optimal strategy when observing a truthful history with $\hat{k}$ revolts is to revolt if willing. If a willing individual observes $h^{\operatorname{tr}}(\hat{k}) \in$ $H^{\operatorname{tr}}(\hat{k})$, then by revolting she generates a history which belongs to $H^{\operatorname{tr}}(\hat{k}+1)$ and by assumption, all subsequent willing citizens will revolt, so the revolution triumphs. Given such a history the unique perfect Bayesian equilibrium strategy is to be truthful, since there is no unilateral profitable deviation.

Note that if we can prove that $\pi_{i}\left(r \mid \tau_{i}=w, h_{i} \in H^{t r}(k), a^{i, *}\right)>\pi_{i}\left(r \mid \tau_{i}=s, h_{i} \in\right.$ $\left.H^{t r}(k), a^{i, *}\right), \forall k>\hat{k}$, for some $\hat{k}$, by induction and applying Lemma (1) recursively, a willing individual chooses to revolt in any best response $\forall k^{\prime} \leq \hat{k}$. It is obvious that if a willing individual observes an element in $H^{t r}(x)$ where $x \geq t-1$, then she will revolt and the revolution triumphs. As a consequence, a willing individual revolts in any truthful history. Note that the first individual who decides is in a truthful history (in fact, any individual who decides without observing any individual who stays at home is trivially in a truthful history). Therefore under social media, in any equilibrium the first individual reveals her type with her action, and so do all the subsequent individuals.

Therefore, under the social media technology, the revolution always succeeds because each willing individual revolts in any Bayesian Nash equilibrium.

19 Clearly, any history with more than $n-W$ "stayings at home" cannot be truthful. 
In order to prove the mass media case, we look for the worst conditions. We consider an extremely low payoff for the case when the revolt does not succeed showing that in this situation, for any positive probability of not having enough willing followers who will revolt, the willing individual will stay at home. We look for the conditions that guarantee that even in the worst case, willing individuals revolt. It proves the conditions under which mass media is sufficient for a successful revolution.

Case $1: t<\left[\frac{n}{n-W+1}\right]+1$.

We split the sequence of individuals into groups with $n-W+1$ individuals in each one and a last group with the reamining individual. Let $g_{i}$ denote each of those groups and $\left\{g_{1}, g_{2}, \ldots, g_{t}, \ldots, g_{\text {integer }\left[\frac{n}{n-W+1}\right]}\right\}$ the sequence of groups. Note that each group $g_{i}$ includes at least 1 willing individual with probability 1 . All the willing individuals in group $g_{t}$ revolt if they observe $t-1$ individuals revolting. For any possible beliefs, individuals in group $g_{t-1}$ revolt if they observe $t-2$ individuals revolting, and by backward induction willing individuals in the group $g_{t-k}$ revolt if they observe at least $t-k-1$ individuals who revolt. Therefore, all the individuals revolt in any Bayesian equilibrium.

Case $2: t \geq\left[\frac{n}{n-W+1}\right]+1$.

We prove that in this circumstance, the following strategy where willing individuals do not revolt over the equilibrium path is a Bayesian equilibrium, with adequate beliefs, for adequate payoffs:

$$
\sigma_{i}=\left\{\begin{array}{l}
r \text { if integer }\left[\frac{n-i}{n-W+1}\right] \geq t-1-\left\{\# a_{j}=r: j<i\right\} \\
s \text { in other case }
\end{array}\right.
$$

where the individual $i$ is in the $i$-th position in the sequence of decision.

The equilibrium where all the willing individuals revolt always exists.

We prove the existence of an equilibrium where nobody revolts. According to the strategy, if the first individual was a willing, she should stay at home as should all the subsequent individuals. In any consistent beliefs, revolting people are willing individuals with probability 1 . For the individuals who do not revolt, according to the strategy, they could be willings as well as unwillings. In the equilibrium beliefs that we construct, the individual assigns a positive probability $\bar{p}$ to having $\min \{i-1, W-1\}$ willing predecessors. Under this belief, the individual is followed by a maximum of $W-i$ willings among her $n-i$ successors. Note that under this belief, there is a positive probability of the first willing successor deciding in position $\min \{\infty, n-(W-i)\}$, where $\infty$ represents the case where it is believed that there is no willing follower. If there is no willing follower, to stay at home is optimal and there is no optimal deviation. If there are willing successors, let be $\breve{p}$ the (strictly positive) probability of the first willing follower being in position $n-(W-i)$. According to the described strategy, note that the willing individual in position $n-(W-i)$ would stay at home even if the individual $i$ revolts. Therefore given that there is a (strictly) positive probability of the revolt being unsuccessful after revolting by the individual $i$, for a payoff of a failed revolt sufficiently low, there is no optimal deviation of staying at home. Therefore, the strategy allow for consistent beliefs that define a Bayesian equilibrium. 
Fig. 2 Decision tree of the social media case in example with $n=4$ and $W=3$

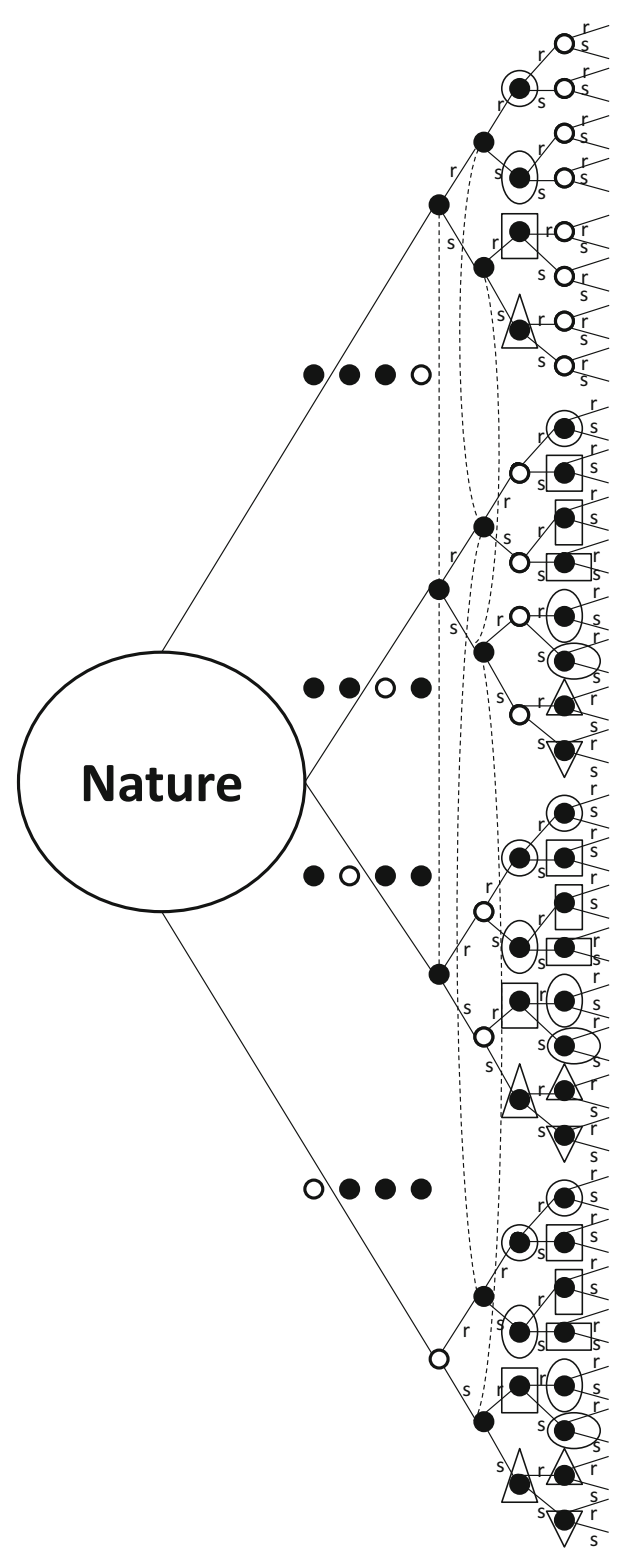

\section{Appendix C}

Figure 2 represents the complete decision tree for the case of social media. Individuals are able to distinguish the sequence of actions but they do not know the type vector. For simplicity, the information nodes that belong to the same information set for individuals in position 3 and 4 have been marked with the same geometrical shape. 


\section{References}

Acemoglu D, Hassan TA, Tahoun A (2014) The power of the street: evidence from Egypt's Arab Spring (No. w20665). National Bureau of Economic Research, Cambridge, MA

Adsera A, Ray D (1998) History and coordination failure. J Econ Growth 3(3):267-276

Angeletos GM, Hellwig C, Pavan A (2007) Dynamic global games of regime change: learning, multiplicity, and the timing of attacks. Econometrica 75(3):711-756

Attia AM, Aziz N, Friedman B, Elhusseiny MF (2011) Commentary: The impact of social networking tools on political change in Egypt's " Revolution 2.0”. Electron Commer Res Appl 10(4):369-374

Azab NA (2012) The role of the internet in shaping the political process in Egypt. Int J E-Polit (IJEP) 3(2):31-51

Banerjee AV (1992) A simple model of herd behavior. Q J Econ 107(3):797-817

Barbera S, Jackson MO (2016) A model of protests, revolution, and information. mimeo, New York

Battaglini, (2017) Public protests and policy making. Q J Econ 132(1):485-549

Bikhchandani S, Hirshleifer D, Welch I (1992) A theory of fads, fashion, custom, and cultural change as informational cascades. J Polit Econ 100(5):992-1026

Bikhchandani S, Hirshleifer D, Welch I (1998) Learning from the behavior of others: conformity, fads, and informational cascades. J Econ Perspect 12(3):151-170

Campante FR, Chor D (2012) Why was the Arab world poised for revolution? Schooling, economic opportunities, and the Arab Spring. J Econ Perspect 26(2):167-187

Carlsson H, Van Damme E (1993) Global games and equilibrium selection. Econometrica 61(5):989-1018

Castells M (2011) The rise of the network society: the information age: economy, society, and culture, vol 1. Wiley, New York

Chen H, Lu YK, Suen W (2016) The power of whispers: a theory of rumor, communication, and revolution. Int Econ Rev 57(1):89-116

Chwe M (2000) Communication and coordination in social networks. Rev Econ Stud 67:1-16

Cooper R, John A (1988) Coordinating coordination failures in Keynesian models. Q J Econ 103(3):441-463

De Mesquita EB (2010) Regime change and revolutionary entrepreneurs. Am Polit Sci Rev 104(03):446466

Dewey T, Kaden J, Marks M, Matsushima S, Zhu B (2012) The impact of social media on social unrest in the Arab Spring. International Policy Program

Edmond C (2013) Information manipulation, coordination, and regime change. Rev Econ Stud 80(4):14221458

Ellis CJ, Fender J (2011) Information cascades and revolutionary regime transitions. Econ J 121(553):763792

Enikolopov R, Makarin A, Petrova M (2016) Social media and protest participation: evidence from Russia. mimeo. Available at SSRN 2696236

Filiu J-P (2011) The Arab revolution: ten lessons from the democratic uprising. Oxford University Press, Oxford

Gentzkow M, Shapiro JM (2011) Ideological segregation online and offline. Q J Econ 126(4):1799-1839

Ghonim W (2012) Revolution 2.0: the power of the people is greater than the people in power-a memoir. Mariner Books, New York

Ginkel J, Smith A (1999) So you say you want a revolution a game theoretic explanation of revolution in repressive regimes. J Conflict Resolut 43(3):291-316

Goldstone J (2001) Toward a fourth generation of revolutionary theory. Annu Rev Polit Sci 4:139-187

Granovetter M (1978) Threshold models of collective behavior. Am J Sociol 83:1420-1443

Gunitsky S (2015) Corrupting the cyber-commons: social media as a tool of autocratic stability. Perspect Polit 13(01):42-54

Howard PN, Duffy A, Freelon D, Hussain MM, Mari W, Maziad M (2011) Opening closed regimes: what was the role of social media during the Arab Spring? Available at SSRN 2595096

Kiss HJ, Rodriguez-Lara I, Rosa-Garcia A (2016) Coordination structures for global threshold games. mimeo, London

Klandermans B (1984) Mobilization and participation: social-psychological expansions of resource mobilization theory. Am Sociol Rev 49(5):583-600

Kuran T (1991) Now out of never: the element of surprise in the East European revolution of 1989. World Polit 44:7-48 
Kuran T (1995) Private truths, public lies: the social consequences of preference falsification. Harvard University Press, Cambridge

Lang JC, De Sterck H (2014) The Arab Spring: a simple compartmental model for the dynamics of a revolution. Math Soc Sci 69:12-21

Lim M (2012) Clicks, cabs, and coffee houses: social media and oppositional movements in Egypt, 20042011. J Commun 62(2):231-248

Lohmann S (1993) A signaling model of informative and manipulative political action. Am Polit Sci Rev 87(2):319-333

Lohmann S (1994a) Information aggregation through costly political action. Am Econ Rev 84(3):518-530

Lohmann S (1994b) The dynamics of informational cascades: the Monday demonstrations in Leipzig, East Germany, 1989-91. World Polit 47(1):42-101

Manacorda M, Tesei A (2016) Liberation technology: mobile phones and political mobilization in Africa. CEP Discussion Paper No 1419

Marzouki Y, Skandrani-Marzouki I, Béjaoui M, Hammoudi H, Bellaj T (2012) The contribution of Facebook to the 2011 Tunisian revolution: a cyberpsychological insight. Cyberpsychol Behav Soc Netw 15(5):237-244

Masiliūnas A (2017) Overcoming coordination failure in a critical mass game: strategic motives and action disclosure. J Econ Behav Organization 139:214-251

Morris S (2014) Coordination, timing and common knowledge. Res Econ 68(4):306-314

Morris S, Shin HS (2003) Global games: theory and applications. In: Dewatripont M, Hansen LP, Turnovsky SJ (eds) Advances in economics and econometrics: theory and applications, eighth world congress, vol 1. Cambridge University Press, Cambridge, UK

Parker E (2014) Social media and the Hong Kong Protests. The New Yorker. Retrieved from http://www. newyorker.com/tech/elements/social-media-hong-kong-protests

Passy F (2003) Social networks matter. But how? In: Diani M, McAdam D (eds) Social movements and networks. Relational approaches to collective action. University Press, Oxford, pp 21-48

Pierskalla JK (2010) Protest, deterrence, and escalation: the strategic calculus of government repression. J Confl Resolut 54(1):117-145

Qin B, Strömberg D, Wu Y (2016) The political economy of social media in China. Working paper

Robinson JA, Acemoglu D (2006) Economic origins of dictatorship and democracy. Cambridge University Press, Cambridge

Schelling TC (1978) Micromotives and macrobehavior. W. W. Norton, New York

Siegel DA (2009) Social networks and collective action. Am J Polit Sci 53(1):122-138

Siegel DA (2011) When does repression work? Collective action in social networks. J Polit 73(04):993-1010

Siegel DA (2013) Social networks and the mass media. Am Polit Sci Rev 107(04):786-805

Steinert-Threlkeld ZC, Mocanu D, Vespignani A, Fowler J (2015) Online social networks and offline protest. EPJ Data Sci 4(1):1

Tufekci Z, Wilson C (2012) Social media and the decision to participate in political protest: observations from Tahrir Square. J Commun 62(2):363-379

Wolfsfeld G, Segev E, Sheafer T (2013) Social media and the Arab spring politics comes first. Int J Press/Polit 18(2):115-137 